\title{
Interprofessionelle Spiritual Care in Palliative Care
}

\author{
Daniel Büchea ${ }^{a}$, Urs Martin Lütolf ${ }^{b}$, Simon Peng-Keller ${ }^{c}$ \\ a Dr. med., Kantonsspital St. Gallen, Zentrumsleiter Palliativzentrum; ' Prof. em. Dr. med., ehem. Ärztlicher Direktor des UniversitätsSpitals Zürich; \\ c Prof. Dr. theol., Professor für Spiritual Care, Universität Zürich
}

\begin{abstract}
Spirituelle Überzeugungen, Praktiken und Erfahrungen können in krankheitsbedingten Belastungssituationen und am Lebensende sowohl eine wichtige Ressource als auch ein Problem darstellen, weshalb sie in die ärztliche Versorgung einzubeziehen sind. Ein kürzlich veröffentlichtes Dokument zu Spiritual Care in Palliative Care regt dazu an, diese Aufgabe vermehrt auch interprofessionell wahrzunehmen.
\end{abstract}

\begin{abstract}
Das Bewusstsein dafür, dass es zur ärztlichen Aufgabe gehören kann, spirituelle Aspekte in die Versorgung einzubeziehen [1, 2], ist in den letzten Jahren in verschiedenen Bereichen der Gesundheitsversorgung gewachsen. Das gilt insbesondere für den Bereich der Palliativmedizin, die 2007 ins MEdBG aufgenommen wurde. Ansätze zur Entwicklung einer ärztlichen Spiritual Care finden sich auch in den neuen Lernzielen fürs Medizinstudium in der Schweiz (PROFILES) sowie in den Weiterbildungskatalogen des SIWF/FMH. Die Umsetzung dieser Zielsetzungen in den ärztlichen Alltag ist allerdings herausfordernd. Eine Studie von Giezedanner et al. [3] zeigte jüngst, dass Schweizer Hausärztinnen und Hausärzte die nicht-somatischen Kompetenzen
\end{abstract}

\section{Résumé}

La prise en charge du spiritual care dans une pratique interprofessionnelle est encore de l'ordre de l'expérience pionnière, dans laquelle les principes empiriques, concepts d'intégration et modèles de pratiques d'excellence doivent être élaborés et contrôlés en fonction du contexte local. Notamment dans les soins palliatifs, on souligne que la mission du corps médical peut englober le côté spirituel des soins. Afin de promouvoir, dans le secteur de la santé en Suisse, une intégration progressive du spiritual care dans la pratique interprofessionnelle, un groupe de travail, créé par l'association palliative ch, a publié un document qui formule les standards de l'assurance qualité et suscite débats et réflexions. Ces directives sont axées sur la question de la compétence professionnelle, mais aussi sur les formes appropriées de communication et de coordination interprofessionnelles. Le groupe de travail pense que le spiritual care ne doit pas être considéré comme un volet complémentaire de la mission médicale, mais bien comme un point qui doit être développé et exploité à titre d'aspect intrinsèque à celle-ci. (spirituelle, kulturelle, ethische, legale) bei der Unterstützung von Sterbenden als fast ebenso wichtig wie die Schmerz- und Symptomkontrolle werten, sich jedoch in diesen Bereichen oft als ungenügend ausgebildet fühlen. 60\% der befragten Ärztinnen und Ärzte sind der Überzeugung, dass ein kompetenter Umgang mit spirituellen Bedürfnissen der Patienten ein wichtiges bis sehr wichtiges Qualitätsmerkmal einer guten palliativmedizinischen Versorgung darstellt. Nur 38\% der Befragten fühlen sich jedoch in dieser Aufgabe sicher.

Was für die Palliative Care in der Schweiz vor 20 Jahren zutraf, gilt heute für die interprofessionelle Spiritual Care: Sie steckt noch in einer Pionierphase, in der die empirischen Grundlagen, integrativen Konzepte und Best-practice-Modelle erarbeitet werden und überprüft werden müssen. Um eine schrittweise Implementierung zu unterstützen und zu begleiten, hat die Schweizerische Gesellschaft für Palliative Medizin, Pflege und Begleitung (palliative ch) 2017 eine interprofessionelle Taskforce Spiritual Care ins Leben gerufen und den Inhaber der Professur für Spiritual Care der Universität Zürich mit deren Leitung beauftragt. Als Instrument für die weitere Entwicklung hat diese Taskforce nun ein Dokument veröffentlicht, das Standards zur Qualitätssicherung ausformuliert und zur Reflexion und Diskussion anregt [4]. Darin wird auch auf verschiedene strittige Fragen eingegangen.

\section{Was bedeutet die "spirituelle Dimension" der Gesundheitsversorgung?}

Die Frage, was die «spirituelle Dimension» der Gesundheitsversorgung genau umfasst, führte bereits 1983 während der 36. Weltgesundheitsversammlung zu intensiven Debatten [5]. Unumstritten ist, dass die Ein- 
führung einer solchen Dimension nur dann sinnvoll ist, wenn sich klar benennen lässt, was sie von der physischen, psychischen und sozialen Dimension unterscheidet. Sie muss konzeptionell hinreichend bestimmt sein und zugleich hinreichend offen für unterschiedliche Überzeugungen. Wie auch die WHODiskussion zeigt, ist die Rede von der spirituellen Dimension der Gesundheitsversorgung eingebettet in ein professionelles Ethos und ist deshalb evaluativ. Nur was zur Förderung von Gesundheit und Lebensqualität von Patientinnen und Patienten beiträgt, ist ärztlicherseits zu fördern. Vor dem Hintergrund der bisherigen Diskussion und vorhandener Konsensdokumente schlägt die Taskforce vor, gesundheitsbezogene Spiritualität zu verstehen als «Verbundenheit einer Person mit dem, was ihr Leben trägt, inspiriert und integriert, sowie die damit verbundenen existenziellen Überzeugungen, Werthaltungen, Erfahrungen und Praktiken, die religiöser oder nichtreligiöser Art sein können» [4].

\section{Wie können die spirituellen Bedürfnisse der Patientinnen und Patienten erfasst werden?}

Zur Erfassung von spirituellen Bedürfnissen von Patientinnen und Patienten wurden in den letzten Jahren verschiedene Assessmentinstrumente entwickelt. $\mathrm{Ob}$ und in welchem Masse sie für die hiesigen klinischen Kontexte geeignet sind, ist allerdings umstritten. $\mathrm{Zu}$ bedenken ist insbesondere, dass es viele Menschen in der Schweiz nicht gewohnt sind, mit Ärztinnen und Ärzten über ihre religiösen und spirituellen Überzeugungen zu sprechen. Es dürfte unter diesen Umständen wenig sinnvoll sein, den Themenbereich in standardisierter Weise anzusprechen. Umgekehrt gilt: Nur was zur Sprache kommen kann, sei es als Belastung oder Ressource, kann in die Behandlung einbezogen werden. Mit Blick auf dieses Dilemma schlägt die Taskforce Spiritual Care u.a. vor, Assessmentinstrumente als Wahrnehmungshilfen zu nutzen und zumindest in dieser Weise konsequent in die Versorgung einzubeziehen.

\section{Professionelle Zuständigkeiten?}

Soll Spiritual Care interprofessionell wahrgenommen werden, müssen die professionellen Zuständigkeiten und die Formen der interprofessionellen Kooperation und Kommunikation geklärt sein. Unbestritten ist, dass Ärztinnen und Ärzte seelsorgliche Fachpersonen nicht ersetzen können. Doch worin genau besteht die ärztliche Aufgabe? Und wo liegen die Grenzen ärztlicher Spiritual Care? Die Taskforce ist der Überzeugung, dass diese nicht als etwas zu betrachten ist, was zusätzlich zu den ärztlichen Aufgaben hinzukommt, sondern als ein Aspekt von diesen erschlossen und entwickelt werden kann. Medizinstudierende müssen deshalb nach den aktuellen Lernzielen lernen, im Rahmen einer Anamnese auch die spirituellen Bedürfnisse von Patientinnen und Patienten zu erfassen. $\mathrm{Zu}$ betonen ist dabei, dass in diesem Bereich von ärztlicher Seite keine

\section{Nur was zur Förderung von Gesundheit und} Lebensqualität von Patientinnen und Patienten beiträgt, ist ärztlicherseits zu fördern.

Lösungen angeboten werden müssen. Es genügt, wenn sie Patientinnen und Patienten vermitteln kann, sie auch in ihren spirituellen Überzeugungen, Haltungen und Erfahrungen ernst zu nehmen.

\section{Rahmenbedingungen zur Umsetzung von Spiritual Care?}

Damit interprofessionelle Spiritual Care sich im hiesigen Gesundheitswesen etablieren kann, braucht es klare und förderliche Rahmenbedingungen. Nur wenn das Angebot von Spiritual Care wie auch die damit verbundenen Aufgaben und Zuständigkeiten in die Versorgungskonzepte einer Institution einbezogen werden und Teil des Qualitätsmanagements sind, kann eine nachhaltige Entwicklung gewährleistet werden. Interprofessionelle Spiritual Care sollte sich an den Qualitätsstandards messen lassen können, die auch für andere Versorgungsbereiche gelten. Das von der Taskforce veröffentlichte Dokument gibt dazu Anstösse und Anregungen, ohne das erst in Entwicklung begriffene Feld umfassend abstecken zu wollen. Denn es lässt sich kaum bestreiten: Der interprofessionelle Lernprozess in diesem Feld hat eben erst begonnen.

\section{Literatur}

1 Peng-Keller S. Ansätze ärztlicher Spiritual Care. Praxis 2017;106(24):1339-43.

2 Peng-Keller S. Spiritual Care als ärztliche Aufgabe? Schweiz Ärzteztg. 2016;97(16):598-600.

3 Giezendanner S, Jung C, Banderet H-R, Otte IC, Gudat H, Haller DM, et al. General practitioners' attitudes towards essential competencies in end-of-life care: A cross-sectional survey. PLoS ONE. 2017;12:e0170168.

4 Peng-Keller S, Mösli P, Aebi R, Barz B, Büche D, Bucher B, et al. Spiritual Care in Palliative Care. Leitlinien zur interprofessionellen Praxis, Bern 2018. Online abrufbar unter: https://www.palliative.ch

5 Peng-Keller S. Spiritual Care im Gesundheitswesens des 20. Jahrhunderts. Vorgeschichte und Hintergründe der WHO-Diskussion um die "spirituelle Dimension", in: Peng-Keller S, Neuhold D (Hg.), Spiritual Care im globalisierten Gesundheitswesen. Historische Hintergründe und aktuelle Entwicklungen, Wissenschaftliche Buchgesellschaft Darmstadt 2019 (im Druck). 